\title{
Infection Control in Medicine with Relevance to Mitophagy and Organ Survival
}

\author{
Ian James Martins ${ }^{1-3 *}$ \\ ${ }^{1}$ Centre of Excellence in Alzheimer's Disease Research and Care, Sarich Neuroscience Research Institute, Edith Cowan University, Australia \\ ${ }^{2}$ School of Psychiatry and Clinical Neurosciences, The University of Western Australia, Australia \\ ${ }^{3}$ McCusker Alzheimer's Research Foundation, Hollywood Medical Centre, Australia \\ *Corresponding Author: Ian James Martins, School of Medical and Health Sciences, Edith Cowan University, Western Australia, \\ Australia.
}

Received: October 11, 2019; Published: October 16, 2019

DOI: 10.31080/ASPS.2019.03.0419

The association between infections, antibiotic use and mitophagy has raised concerns with relevance to the doses of antibiotics in mitochondrial dynamics and antibiotic induced mitochondrial apoptosis in these chronic diseases with irreversible cell death associated antibiotics and multisystem organ disease. The anti-aging gene Sirt 1 is a NAD+ dependent class III histone deacetylase activity involved in the regulation of metabolic activity, insulin resistance and inflammatory processes and is now important to drug therapy and metabolism with relevance to prevention of drugdrug interactions. Sirt 1 is involved in drug metabolism and linked to hepatic glucose, fatty acid and caffeine metabolism. Nutritional interventions that regulate Sirt 1 have become critical to maintain antibiotic use and drug therapy with relevance to non alcoholic fatty liver disease (NAFLD), cardiovascular disease and neurodegeneration. Consumption activators of Sirt 1 (magnesium, leucine, alpha-lipoic acid, pyruvic acid, resveratrol) are important to maintain antibiotic use and drug therapy/metabolism.

In the developing world Sirt 1 may be repressed with relevance to defective xenobiotic metabolism and mitophagy. Increased plasma levels of bacterial lipopolysaccharides (LPS) in developing world individuals may repress Sirt 1 and inactivate antibiotic and drug therapy. The use of Indian spices and antibiotic use need to be carefully controlled with relevance to effective mitochondrial dynamics and to maintain mitochondrial biogenesis. Infection prevention and control by antibiotics may become ineffective with diet, drug and inhibitors (drug-drug interactions) that may disrupt Sirt 1 with relevance to autoimmune disease and rejection of the pancreas, thyroid, kidneys, liver, heart and brain. Magnesium defi- ciency may inactivate antibiotic and drug therapy with irreversible cell death associated antibiotic inactivation, polypharmacy and organ suicide [1-13].

\section{Bibliography}

1. IJ Martins. "Antibiotic use and Nuclear Receptor Inactivation linked to Mitophagy in Diabetes and Chronic Diseases".Journal of Diabetes and Clinical Studies 2 (2018): 1-3.

2. Increased Risk for Obesity and Diabetes with Neurodegeneration in Developing Countries. Top 10 Contribution on Genetics. Chapter 1, E Book (2018).

3. Ian James Martins. "Anti-Aging Gene linked to Appetite Regulation Determines Longevity in Humans and Animals". International Journal of Aging Research 1 (2018): 1-4.

4. IJ Martins. "Bacterial Lipopolysaccharides and Neuron Toxicity in Neurodegenerative Diseases". Neurology Research and Surgery 1 (2018): 1-3.

5. IJ Martins. "The Future of Genomic Medicine Involves the Maintenance of Sirtuin 1 in Global Populations". International Journal of Molecular Biology 2 (2017): 00013.

6. IJ Martins. "Diet, Drug and Inhibitor Therapy Prevent Toxic Protein Aggregation in Various Species". Acta Scientific Nutritional Health 2 (2018): 01-03.

7. IJ Martins. "Indian Spices and Biotherapeutics in Health and Chronic Disease”. Health 10 (2018): 374-380. 
8. IJ Martins. "Sirtuin 1, a Diagnostic Protein Marker and its Relevance to Chronic Disease and Therapeutic Drug Interventions". EC Pharmacology and Toxicology 6.4 (2018): 209-215.

9. IJ Martins. "Indian Spices and Unhealthy Diets interfere with Drug Therapy in Diabetes and Neurodegenerative Diseases". NAPDD 3 (2018): 555616.

10. IJ Martins. "Antimicrobial Drugs and Bacterial Amyloid Peptide Induce Toxic Manifestations in Chronic Diseases". EC Pharmacology and Toxicology 6.1 (2018): 01-04.

11. IJ Martins. "Antibiotic Resistance Involves Antimicrobial Inactivation in Global Communities". SAJ Pharmacy and Pharmacology 2 (2017): 102.

12. IJ Martins. "Drug-Drug Interactions with Relevance to Drug Induced Mitochondrial Toxicity and Accelerated Global Chronic Diseases". EC Pharmacology and Toxicology 3.1 (2017): 18-21.

13. IJ Martins. "Magnesium Therapy Prevents Senescence with the Reversal of Diabetes and Alzheimer's Disease". Health 8 (2016): 694-710.

Volume 2 Issue 11 November 2019

(C) All rights are reserved by Ian James Martins. 